\title{
Ostéonécrose Cortico-induite Multifocale au Cours d'une Leucémie Aigue Lymphoblastique: A Propos d'un Cas
}

Matrane Wafaa, Bendari Mounia, Nadif Hajar, Service de Radiologie, Casablanca, Maroc

Quessar Asmaa, Khoubila Nisrine,

Service d'Hématologie et d'Oncologie Pédiatrique, Casablanca, Maroc

\section{Résumé}

Les corticostéroïdes oraux sont couramment utilisés en hématologie, en particulier dans le traitement des leucémies aiguës et des lymphomes. L'ostéonécrose est une complication significative et invalidante de la corticothérapie. Les patients du service d'hématologie reçoivent des thérapies combinées à base de chimiothérapies et de corticoïdes. Ils sont ainsi à risque élevé de développer une ostéonécrose avasculaire, qui est malheureusement le plus souvent diagnostiquer aux stades avancés de la maladie. Par conséquent, les cliniciens doivent être conscients du potentiel risque de cette complication et de l'importance d'un diagnostic précoce et d'un traitement adéquat afin d'améliorer le pronostic fonctionnel de ces patients qui ont survécu à leur maladie cancéreuse. Nous rapportons le cas d'un jeune adulte atteint de leucémie lymphoblastique aiguë, traité par une combinaison de corticoïdes et de chimiothérapie, compliquée d'une ostéonécrose multifocale, et discutons brièvement cette complication rare mais redoutable à la lumière de la littérature existante.

Mots-clés: Leucémie aigue lymphoblastique, Corticostéroïdes, Ostéonécrose multifocale, Adulte jeune 


\title{
Multifocal Cortisteroid-induced Osteonecrosis in Acute Lymphoblastic Leukemia: A Case Report
}

\author{
Matrane Wafaa, \\ Bendari Mounia, \\ Nadif Hajar, \\ Service de Radiologie, Casablanca, Maroc \\ Quessar Asmaa, \\ Khoubila Nisrine, \\ Hematology and Pediatric Oncology department, Casablanca, Morocco
}

\begin{abstract}
Osteonecrosis is recognized as one of the most common therapyrelated side effect in patients with acute lymphoblastic leukemia. Long continuous use of corticosteroid during all therapy is the major contributor to the development of osteonecrosis. To reduce osteonecrosis which is associated with debilitating long-term effects, steroid prescription should be rational. Patients should be diagnosed in early asymptomatic stages of osteonecrosis by MRI to improve prediction, management, and to prevent functional impairment. This paper focuses on reporting a case of a young adult treated with a combination of high dose corticosteroids and multi-agent chemotherapy for acute lymphoblastic leukemia. It was complicated by development of a multifocal osteonecrosis. This rare but serious complication was also briefly discussed in light of the existing literature.
\end{abstract}

Keywords: Acute lymphoblastic leukemia, Corticosteroids, Multifocal osteonecrosis, Young adult

\section{Introduction}

L'Ostéonécrose aseptique (ONA) est une pathologie invalidante qui touche principalement l'adule jeune, en pleine activité (Liu et al., 2017). Elle est au moins deux fois plus fréquente chez les hommes que chez les femmes (Hernigou, 2006) et elle a été reconnue comme étant l'une des complications les plus significatives des traitements anti-leucémiques (Kunstreich et al., 2016), ayant un pronostic fonctionnel parfois dramatique (Beguin et al., 1999; Riccio et el., 2016). L'ostéonécrose a également été rapportée chez des patients qui ont subi une greffe de cellules souches hématopoïétiques, et a été 
attribuée à l'utilisation de corticostéroïdes pour contrôler la réaction du greffon contre l'hôte (Karimova et al., 2017). Les nécroses multifocales sont relativement fréquentes et atteignent préférentiellement, la hanche, l'épaule et le genou (Hernigou, 2006). Cette pathologie évolue progressivement avec des épisodes de poussées douloureuses, mécaniques pouvant devenir permanentes (Lafforgue, 2009; Mayer et al., 2013).

Son diagnostic repose exclusivement sur l'imagerie et l'IRM reste à l'heure actuelle l'examen de référence pour dépister les atteintes précoces. La prise en charge de l'ONA dépend essentiellement du stade diagnostic de la maladie, de l'état général et de l'âge du patient (Loukili et al., 2014). Nous rapportons le cas d'un jeune patient traité pour leucémie aigue lymphoblastique, compliquée d'ostéonécrose multifocale touchant les deux têtes fémorales et la tête humérale droite, à travers lequel nous insisterons sur le diagnostic et la prise en charge précoce de cette complication rare mais redoutable des traitements anti-leucémiques.

\section{Observation}

Nous rapportons le cas d'un jeune patient âgé de 19 ans, sans antécédents pathologiques particuliers, sans habitudes toxiques et sans comorbidités. Le patient était admis en consultation d'hématologie pour un syndrome anémique fait de pâleur cutanéomuqueuse, d'asthénie et pour un syndrome hémorragique fait de purpura ecchymotique au niveau des membres inférieurs. Suite auxquelles les investigations biologique, cytologique, immunophénotypage et cytogénétique ont conclu à une leucémie aigue lymphoblastique (LAL) de phénotype B, initialement non hyperleucocytaire, avec au caryotype une hypotryploidie. La recherche du réarrangement $\mathrm{BCR} / \mathrm{ABL}$ était négative. Le patient était inclus au protocole Marocain des LAL (Protocole MARALL 2006, Risque élevé). Ce traitement est étalé sur deux ans et 10 mois. Or, cette durée peut varier en cas de survenue de complications au cours du traitement, nécessitant des arrêts provisoires des cures. Le protocole commence par 7 jours de corticothérapie et d'une ponction lombaire (Pl) triple (préphase), ensuite une phase d'induction composée de Prédnisone, Vincristine, Asparaginase, Daunorubicine et de Pl triple, puis une phase de consolidation comportant du Mercaptopurine, Cyclophosphamide, Aracytine et $\mathrm{Pl}$ triple pour la première partie et du Prednisone, Mercaptopurine, Vincristine, Méthotrexate haute dose et de Pl triple pour la deuxième partie de la consolidation. S'ensuit une première intensification, phase de chimiothérapie lourde, similaire à l'induction. Puis une interphase, une deuxième intensification et enfin un traitement d'entretien de deux ans à base de Vincristine, Déxamethasone, Méthotrexate faible dose, Mercaptopurine. 
Cinq mois après le début du traitement de sa leucémie, le jeune patient s'est présenté au service pour des douleurs osseuses d'allure mécanique des membres inférieures principalement au niveau des racines des cuisses associées à une boiterie. L'examen clinique du malade a retrouvé une limitation douloureuse des mouvements articulaires des deux hanches et ne retrouvait pas de syndrome tumoral associé. L'hypothèse de rechute de la maladie était éliminée par la réalisation d'un myélogramme qui a retrouvé une moelle riche non envahie plaidant en faveur de la rémission médullaire maintenue. Les radiographies standards du bassin (Figure 1) révélaient une perte de la sphéricité des deux têtes fémorales avec aspect irrégulier de ses contours, avec pincement articulaire prédominant à droite et l'imagerie par résonance magnétique du bassin (IRM) (Figure 2) a confirmé le diagnostic d'ostéonécrose aseptique bilatérales des têtes fémorales par la présence d'un aspect aplati des deux têtes fémorales et d'un liseré en hyposignal Tl et Stir délimitant des zones hypersignal T1, s'effacant en Stir, en rapport probablement avec des séquestres de signal graisseux. Il s'y associe de multiples lésions en plage métaphysaires et diaphysaires fémorales bilatérales, des ailerons sacrés et de l'aile iliaque gauche. Ces lésions sont en hypersignal T1, effacé sur la séquence Stir et sont entouré d'un halo périphérique en hyposignal sur la séquence T1 et en hypersignal sur la séquence Stir.

Le patient a poursuivi son protocole de chimiothérapie sans corticostéroïdes, cependant le tableau clinique s'est compliqué par l'accentuation des douleurs avec marche à l'aide de béquilles. Le patient avait bénéficié d'un traitement local constitué d'une prothèse totale de la hanche droite. Les suites post opératoires étaient simples sans incident (Figure 3). L'amélioration fonctionnelle et la mobilité était très appréciable. En attendant de bénéficier d'une arthroplastie gauche, deux mois plus tard le patient présentait une légère douleur de l'épaule droite sans impotence fonctionnelle, pour laquelle une IRM de l'épaule (Figure 4) a été rapidement de mise, objectivant une ostéonécrose de la tête humérale droite avec séquestre de signal graisseux (Type A) compliquée d'un tassement sous chondral et un infarctus diaphysaire huméral proximal.

Le patient est actuellement en rémission complète de sa leucémie, suivi régulièrement en consultation d'hématologie et de traumatologie, et il est programmé prochainement pour une PTH gauche. Il n'a plus de douleurs et marche sans appui. 


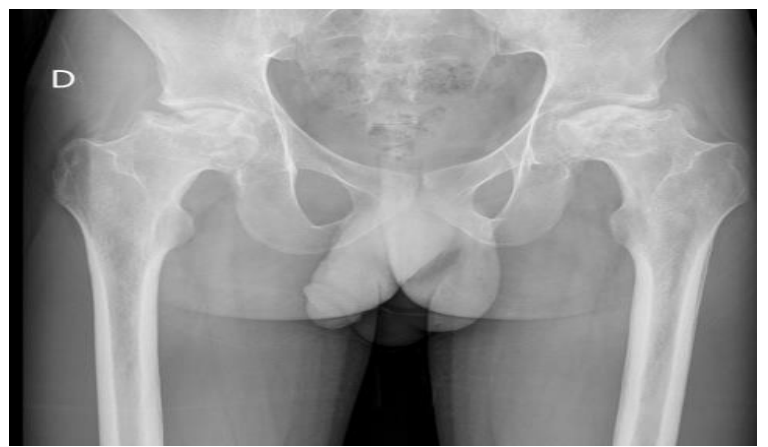

Figure 1. Radiographie standard du bassin face Perte de la sphéricité des deux têtes fémorales avec aspect irrégulier de ses contours et pincement articulaire prédominant à droit
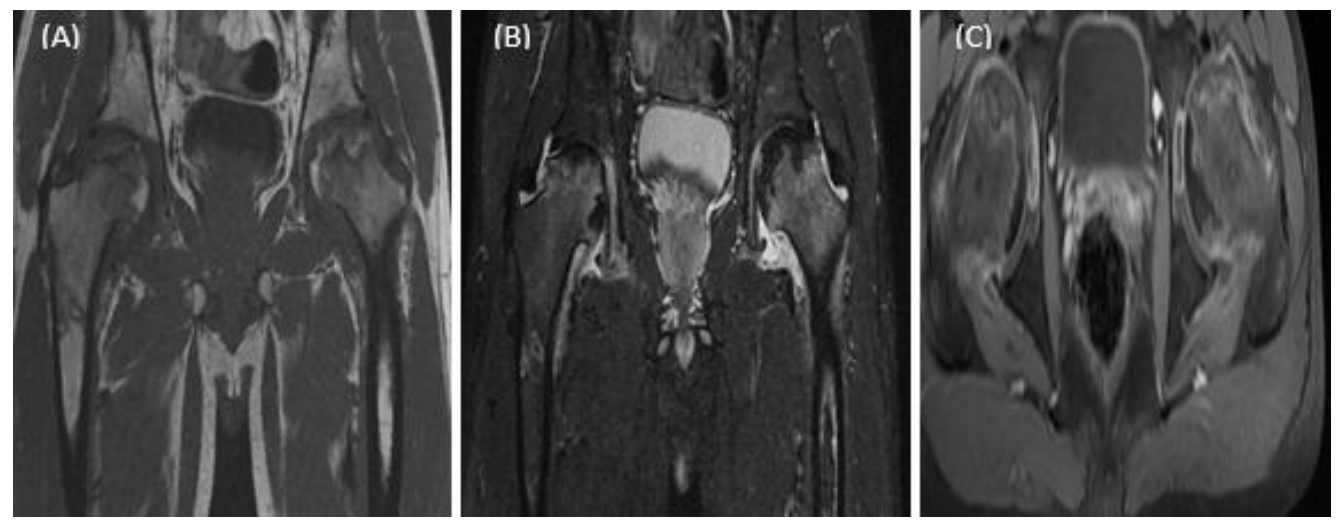

Figure 2. IRM du bassin en coupe coronale T1(A), coronale T2 Fatsat (B), et axiale après injection de gadolinium $(\mathrm{C})$

Ostéonécrose de deux têtes fémorales avec séquestre osseux de signal graisseux (Type A selon la classification de Mitchell). Plage en hypersignal T1, hyposignal T2 Fatsat hétérogène, bien limitée, cernée par une ligne en hypersignal, non rehaussée après injection de gadolinium, doublée d'un hypersignal T2 en rapport avec le liseré de démarcation.

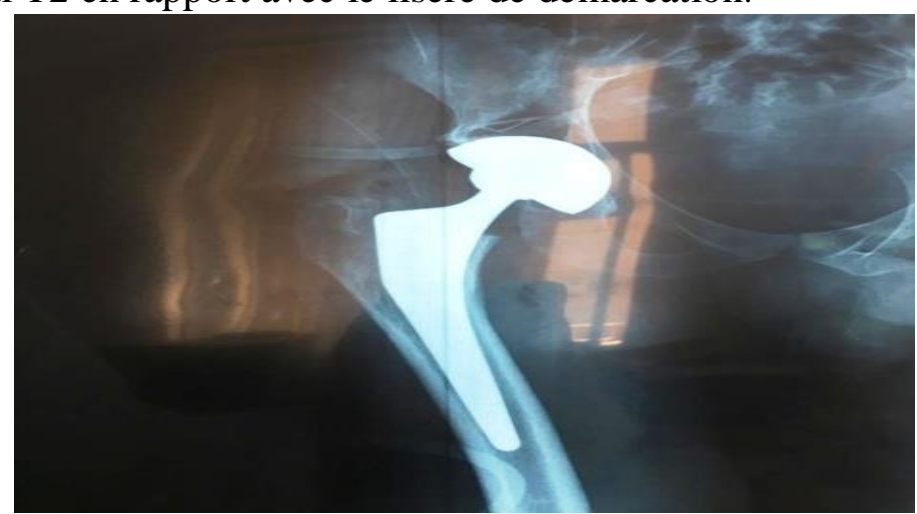

Figure 3. Radiographie standard profil de la hanche droite en postopératoire Prothèse totale de la hanche droite 


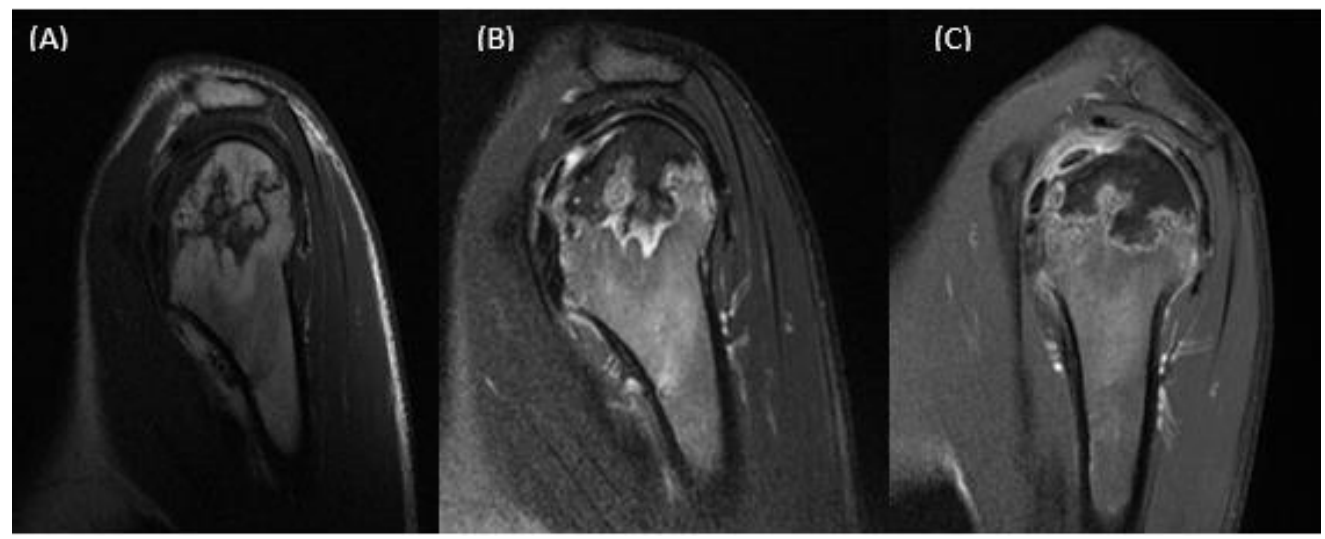

Figure 4. IRM de la tête humérale droite en coupe sagittale T1(A), Fatsat (B) et T1 après injection de gadolinium (C)

Ostéonécrose de la tête humérale droite avec séquestre osseux de signal graisseux (Type A selon la classification de Mitchell). Plage en hypersignal T1, hyposignal T2 Fatsat hétérogène, bien limitée, cernée par une ligne en hypersignal, non rehaussée après injection de gadolinium, doublée d'un hypersignal $\mathrm{T} 2$ en rapport avec le liseré de démarcation.

\section{Discussion}

L'Ostéonécrose aseptique (ONA) correspond à la mort de l'ensemble des composants tissulaires de l'os (moelle hématopoïétique et tissu minéralisé), avec persistance uniquement d'une trame osseuse déshabitée (Lafforgue, 2009). Ce n'est pas une maladie spécifique, mais c'est plutôt le résultat de l'intrication de diverses conditions pathologiques dont la majorité aboutit à l'altération de la circulation sanguine intraosseuse (Hernigou, 2006). Elle peut être idiopathique dans environ $40 \%$ des cas ou secondaire à un ou plusieurs facteurs de risques comme le traumatisme, la corticothérapie, certains toxiques notamment l'alcool, la maladie des caissons hyperbares, la drépanocytose, la maladie de gaucher, les maladies systémiques ou autoimmunes et les chimiothérapies... (Loukili et al., 2014; Abrisham et al., 2013).

La fréquence de survenue d'ONA chez les enfants et les adolescents traités pour LAL est environ de l'ordre de 9\% (Mayer et al., 2013). Elle est de plus en plus attribuée aux traitements intensifs aux corticostérö̈des (Karimova et al., 2017). En dehors des stéroïdes et des facteurs de risque sus cités le Méthotrexate utilisé à forte dose dans notre protocole a été également rapportées dans la littérature (Beguin et al., 1999).

La tête fémorale représente le siège électif de l'ostéonécrose en raison de son type de vascularisation artérielle terminale, où toute modification de la perfusion sanguine n'est pas compensée conduisant ainsi à une disparité entre les besoins en oxygène et le défaut d'apport par la circulation locale (Riccio et 
al., 2016; Ghebhard et al., 2001; Mirzai et al., 1999). Elle est bilatérale dans $70 \%$ des cas et en terme de fréquence, la tête humérale représente la seconde localisation préférentielle après la hanche. Les localisations multiples sont fréquemment méconnues en raison de la pauvreté et le retard d'apparition de la symptomatologie clinique et des anomalies radiologiques (Hernigou, 2006). Jusqu'à présent, les mécanismes par lesquels les glucocorticoïdes induisent une nécrose avasculaire ne sont pas complètement compris. Les hypothèses comprennent, l'augmentation de la lipogenèse, hypercoagulabilité et diminution de l'activité ostéoblastique (Beguin et al., 1999).

Quel que soit l'étiologie de l'ONA, le diagnostic au stade de début peut être difficile en raison de l'absence du parallélisme clinico-radiologique, de l'absence de spécificité des signes cliniques et la discrétion des signes radiologiques. Au début, la maladie est insidieuse et les signes d'appel sont inconstants et seraient en fonction du stade de la nécrose (Loukili et al., 2014). Cliniquement elle se manifeste par l'apparition brutale d'une douleur, mécanique, évoluant progressivement vers une gêne fonctionnelle, et mettant en jeu le pronostic fonctionnel de l'articulation en absence de diagnostic et de traitement précoce (Lafforgue, 2009; Mayer et al., 2013).

La radiographie standard est l'examen réalisé en 1ère intention, et le plus fiable pour rechercher le classique aspect de " coquille d'œuf ", cependant elle est le plus souvent d'aspect normal et ne détecte jamais la maladie au stade de début. Les signes radiologiques les plus précoces ne sont pas spécifiques, à type de simples plages de clarté et/ou de condensation hétérogènes (Loukili et al., 2014; Hernigou, 2006). Donc le recours à l'IRM dans les cas débutants paraît nécessaire. L'avantage de l'IRM est également de dépister une éventuelle ostéonécrose sur la hanche controlatérale qui peut être asymptomatique. Le signe le plus constamment décrit est un liseré de démarcation en hyposignal sur les séquences pondérées en $\mathrm{T} 1$ et un aspect appelé double ligne de Mitchell, correspondant à hyposignal en périphérie sur lequel s'accole en dedans une bande en hypersignal (Lafforgue, 2009). La Scintigraphie osseuse montre une hyperfixation précoce non spécifique et la tomodensitométrie (TDM) n'est pas très performante pour le diagnostic précoce, cependant il met en évidence en coupes transversales la dissection sous chondrale. Ces examens, sont utiles au bilan de la maladie et n'ont d'intérêt diagnostic qu'en cas d'impossibilité à réaliser une IRM qui a la meilleure sensibilité et spécificité pour le diagnostic d'ostéonécrose (Lafforgue, 2009; Loukili et al., 2014).

La prise en charge de cette pathologie a pour but de rétablir la fonction articulaire et d'obtenir une articulation mobile, stable et indolore. Elle est essentiellement orthopédique allant du forage simple, jusqu'à l'arthroplastie (Kleisch et al., 2015; Mukisi Makaza et al., 2011). 
Le traitement médical ne permet pas de limiter la progression de la maladie, il est de mise dans l'attente d'une intervention chirurgicale ou en cas de contre-indications à l'anesthésie ou lorsque l'intervention pourrait être trop risquée. Il repose essentiellement sur le traitement de la douleur et sur la mise en décharge de l'articulation (Hernigou, 2006).

A un stade avancé, le forage garde uniquement un intérêt antalgique mais ne suffit plus à empêcher la progression des lésions. Une chirurgie conservatrice serait idéalement à envisager pour retarder au maximum l'heure de l'arthroplastie chez ces jeunes. Au stade arthrosique, le remplacement prothétique devient indispensable (Beguin et al., 1999).

Les patients recevant des corticostéroïdes par voie orale doivent être avertis du risque potentiel de nécrose d'une ou plusieurs articulations. Chez ce patient la survenue d'ONA a été attribuée à l'usage de corticoïdes qui sont utilisés avec des fortes doses et pendant de longues périodes dans le protocole MARALL. Le patient n'avait pas de facteurs de risque autre que la corticothérapie notamment pas de notion de traumatisme, pas d'habitudes toxiques ni d'autres pathologies associés à la LAL. La notion de douleurs articulaires ou osseuses antérieures au traitement qui sont parfois le mode de révélations de certaines LAL n'était pas rapporté par le malade. Il faut donc garder cette complication à l'esprit à chaque fois qu'un patient développe soudainement des douleurs articulaires après une corticothérapie. La population à risque doit être surveillée de près et doit être référée à des orthopédistes dès la suspicion de la maladie pour un diagnostic et un traitement précoce afin de préserver l'articulation. De ce fait, les cliniciens doivent veiller à une utilisation rationnelle des corticostéroïdes avec respect des indications et des posologies.

\section{Conclusion}

Les succès achevés dans le traitement des LAL peuvent s'expliquer en partie par l'intensification thérapeutique, qui augmente toutefois le risque des effets secondaires qu'il faut prévenir et guetter. L'ONA est bien connue pour être l'une des complications rares mais redoutables de la corticothérapie. Étant donné qu'elle touche principalement une population jeune, tout doit être mis en œuvre pour préserver l'articulation et retarder le remplacement prothétique. Le meilleur facteur pronostique du traitement reste sans doute la précocité du diagnostic et L'IRM trouve sa meilleure indication dans le diagnostic précoce et différentiel, ainsi que dans la classification de l'ostéonécrose.

\section{Conflits d'intérêts}

Les auteurs ne déclarent aucun conflit d'intérêts. 


\section{Contributions des auteurs}

Tous les auteurs ont lu et approuvé la version finale du manuscrit.

\section{References:}

1. Abrisham, SM., Hajiesmaeili, MR., Soleimani, H., \& Pahlavanhosseini, H. (2003). Efficacy of core decompression of femoral head to treat avascular necrosis in intravenous drug users. Acta Med Iran; 51 (4):250-3.

2. Beguin, A., Bertrand, Y., Ourcival, S., Foray, P., Mane, A M., \& Philippe, N. (1999). Ostéonécrose aseptique de la fémorale au cours du traitement des leucémies aigues lymphoblastiques de l'enfant. Arch Pediatr; 6(9): 971-4.

3. Gebhard, K L. \& Maibach, H I. (2001). Relationship Between Systemic Corticosteroids and Osteonecrosis. Am J Clin Dermatol; 2(6):377-88.

4. Hernigou, P. (2006). Ostéonécroses non traumatiques des épiphyses de l'adulte. EMC (Elsevier SAS, Paris), Appareil locomoteur, 14-028-A10.

5. Kunstreich, M., Kummer, S., Laws, H-J., Borkhardt, A., \& Kuhlen, M. (2016). Osteonecrosis in children with acute lymphoblastic leukemia. Haematologica; 101(11): 1295-1305.

6. Karimova, E J., Rai, S N., Howard, S C., Neel, M., Britton, L., Pui, C H., \& Kaste, S C. (2017). Femoral Head Osteonecrosis in Pediatric and Young Adult Patients With Leukemia or Lymphoma. J Clin Oncol; 25: 1525-1531.

7. Kleisch, A-M., Zulfiqar, A-A., \& Novella1, J-L. (2015). Ostéonécrose aseptique de la tête fémorale : un diagnostic à ne pas méconnaître. Rev Geriatr; 40(4): 237-8.

8. Lafforgue, P. (2009). Ostéonécrose de la tête fémorale. Rev Rhumatol; 76(2):166-172.

9. Loukil, H., Frikha, F., Snoussi, M., Ben Salah, R., \& Bahloul, Z. (2014). Ostéonécrose aseptique de la tête fémorale chez une patiente atteinte d'artérite de Takayasu. Pan Afr Med J; 18: 203.

10. Liu, LH., Zhang, QY., Sun, W., Li, ZR., \& Gao, FQ. (2017). Corticosteroid induced Osteonecrosis of the Femoral Head: Detection, Diagnosis, and Treatment in Earlier Stages. Chin Med J; 130: 26017.

11. Mukisi-Mukaza, M., Saint-Martin, C., Étienne-Julan, M., Donkerwolcke, M., Burny, ME., \& Burny, F. (2011). Risk factors and impact of orthopaedic monitoring on the outcome of avascular necrosis of the femoral head in adults with sickle cell disease: 215 patients case study with control group. Orthop Traumatol Surg Res; 97(8): 814-20. 
12. Mayer, S W., Mayer, B K., Mack Aldridge, J., Urbaniak, J R., Fitch, R D., \& Lark, R K. (2013). Osteonecrosis of the femoral head in childhood malignancy. J Child Orthop; 7(2): 111-16.

13. Mirzai, R., Chang, C., Greenspan, A., \& Gershwin, ME. (1999). The pathogenesis of osteonecrosis and the relationship to corticosteroids. J Asthma; 36(1):77-95.

14. Riccio, I., Pota, E., Marcarelli, M., Affinita, M C., Pinto, D D., \& Indolfi $\mathrm{C}$ et al. (2016). Osteonecrosis as a complication in pediatric patients with acute lymphoblastic leukemia. Pediatr Med Chir; 38:118. 